\title{
SLIPPERY AND NEVER WET
}

¿ Matilda Backholm, Jaakko V. I. Timonen ${ }^{\star}$, and Robin H. A. Ras ${ }^{\star}$ - Dol: https://doi.org/10.1051/epn/2017505 I Department of Applied Physics - Aalto University - P.O. Box 15100, 02150 Espoo, Finland

n * Email: jaakko.timonen@aalto.fi, robin.ras@aalto.fi

\section{Superhydrophobic surfaces let water droplets roll off with low friction and falling} droplets rebound, leaving the surfaces completely dry. Such extremely water repellent surfaces are found in nature on lotus leaves, the legs of water striders and feather coatings of birds, and portray a beautiful example of ingenious biological design. They provide an exciting research avenue for physicists and materials scientists aspiring to understand and mimic nature.

\section{A physicist's approach}

to superhydrophobicity

Superhydrophobicity, that is the extreme fear of water, is a fascinating surface property found in nature on many plants and insects. The strong water repellency is often crucial for surviving in harsh conditions: the superhy- drophobic surface on submerged insects, for example, traps small air pockets on their bodies that act as an external lung to allow gas exchange and breathing underwater. Barthlott and Neinhuis realized in 1997 that the intriguing self-cleaning nature of a lotus leaf arises from the combination of surface microroughness and a low-surface-energy wax nanocrystal coating (Fig. 1a) [1]. This discovery opened an exciting avenue for scientists aspiring to mimic the designs found in nature to manufacture artificial superhydrophobic substrates. 
The grand goal is to produce a world of self-cleaning, non-wetting, anti-fouling, flow-enhancing, anti-icing, and anti-fogging materials, just to name a few examples. Currently, the major bottleneck towards commercialization of superhydrophobic surfaces is the fragility of the micro/nano-structured substrate. A damaged surface region will make drops stick to the material and vastly degrade the final product. Intense research is currently being performed to create new, mechanically robust alternatives [2-3].

Today, the most common physical model of a superhydrophobic surface is made of an array of micron-sized pillars coated with a hydrophobic film (Fig. 1b). When a water droplet is placed on the surface, air becomes trapped within the micropillared structure (Fig. 1c-d). The droplet thus rests mostly on a cushion of air, visible as a thin ray of light between the droplet and the solid substrate. This is called the Cassie wetting state and allows for very high contact angles $\left(\theta>150^{\circ}\right)$ and extremely low droplet friction and adhesion. Recently, beautiful sideview projections utilizing confocal microscopy from the Butt group provided a high-resolution view of the contact line of a droplet in the Cassie state (Fig. 1e) [4]. Detailed information on the microscopic motion of the contact line was also gained, shedding light on the fundamentals
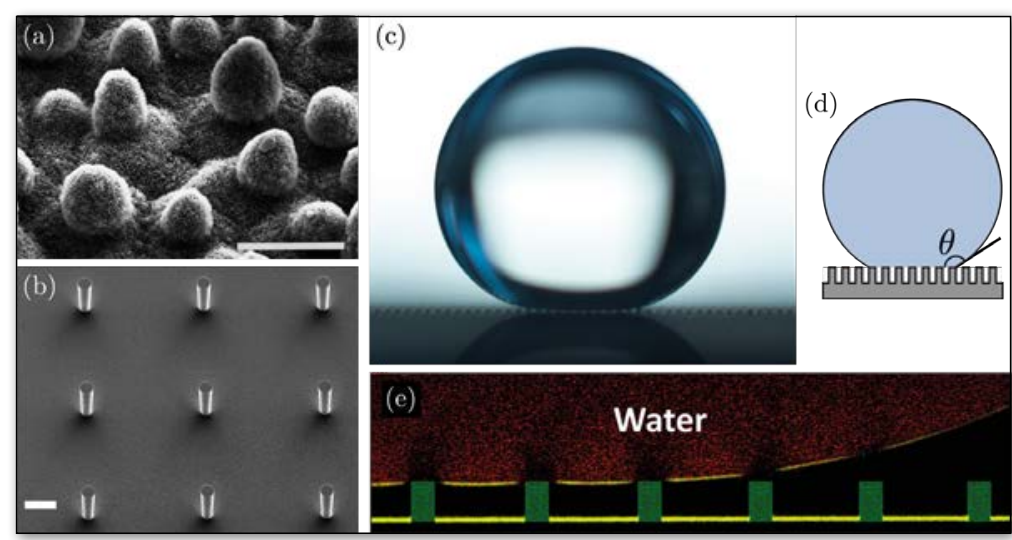

$\triangle$ FIG. 1: The physical model of a superhydrophobic surface. (a) Scanning electron microscopy (SEM) image of the surface of a sacred lotus (Nelumbo nucifera) leaf (scale bar $20 \mu \mathrm{m}$ ). Picture adapted from [1]. (b) SEM image of an artificial micropillared surface (scale bar $20 \mu \mathrm{m}$ ). Image by Anas AI-Azawi. (c-d) Photo and schematic illustration of a millimetric water droplet in the Cassie state on a micropillared superhydrophobic surface. An air layer is trapped within the microstructure beneath the droplet, rendering a very high contact angle $\theta$. Photo by Mika Latikka. (e) Confocal microscopy image of the three-phase contact line of the drop (pillar spacing $30 \mu \mathrm{m}$ for scale). Picture from [4].

4

\section{The grand goal is to produce a world of self-cleaning, non-wetting, anti-fouling, flow-enhancing, anti-icing, and anti-fogging materials.}

v FIG. 2: Transitions between wetting states. (a) Schematic illustrations of irreversible and reversible wetting transitions on a single (left) and dual-level (right) topography, respectively. (b) The compression (plate/substrate gap denoted by z, plate direction by arrow) of a droplet on a micropillared superhydrophobic surface with single (left) and dual-level (right) topography. In the former, the droplet irreversibly undergoes a transition to the Wenzel state, whereas the latter allows for a reversible transition. The two first, central images (black boxes) show experiments on a dual-level surface, but also capture the initial stages on the single-level surface. The Cassie state is noted by the bright region between the drop and the surface. Pictures in (a) adapted from [6] and (b) adapted from [7].

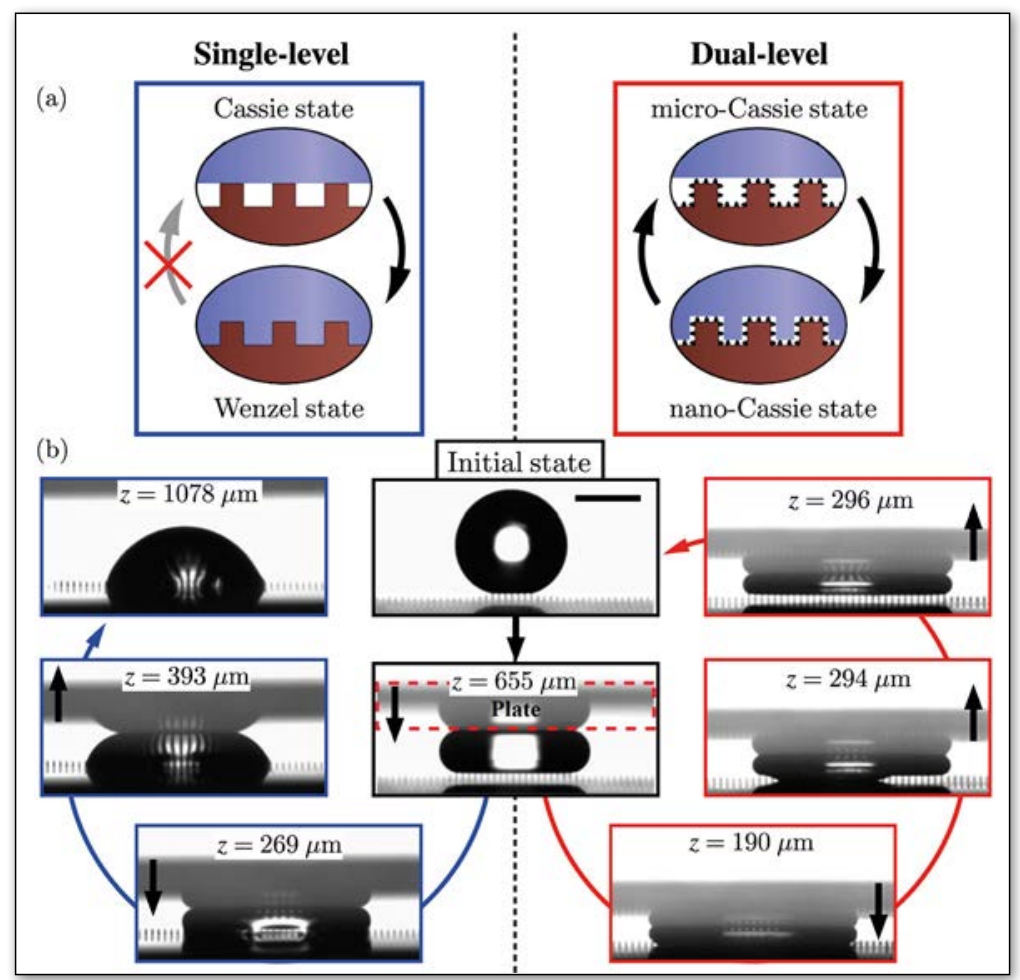


behind the almost unhindered dynamics of droplets on these surfaces.

Reversible wetting transitions

The Cassie state is crucial for superhydrophobicity to arise. Unfortunately, pressure fluctuations, such as from an impacting droplet, can collapse the air cushion between the water droplet and the solid substrate, resulting in water to wet the surface completely, called Wenzel state (Fig. 2 left). This wetting transition is

\section{The droplet thus rests mostly on a cushion of air, visible as a thin ray of light between the droplet and the solid substrate.}

v FIG. 3: Bouncing droplets. (a) Conventional axisymmetric recoil of a water droplet hitting a superhydrophobic silicon surface (top: side view, bottom: top view). (b) Non-axisymmetric recoil of droplet on a macroscale textured surface (top: side view, bottom: top view). Pictures in (a-b) adapted from [8]. (c) Side view of typical bouncing (top) and unconventional pancake bouncing (bottom) of droplet on surface with tapered posts covered by nanoflowers. Picture from [9].
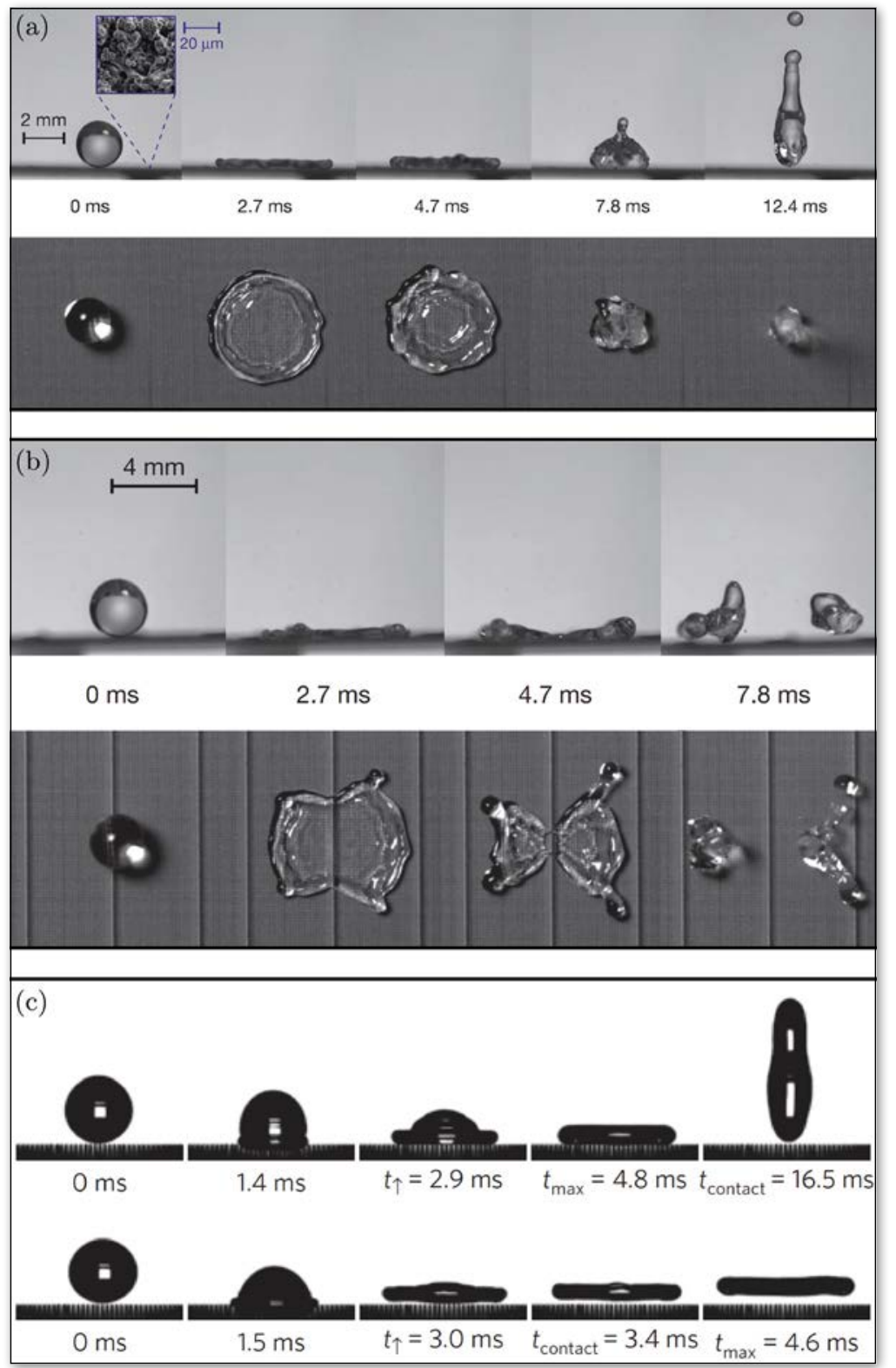

usually irreversible and the droplet becomes pinned to the surface. To avoid the Wenzel state, we took guidance from aquatic insects such as the water boatman Notonecta glauca, that can breathe underwater thanks to the air retention feature of their superhydrophobic hairy exoskeleton. Barthlott et al. observed that while aquatic insects with long hair can support a large volume of air, insects with small hairy structures had supreme lifetime of the air film, arguing that downward progression of the air-water interface is hindered due to the fact that it costs more energy to displace an interface with a smaller radius of curvature [5]. We introduced a dual-level topography by coating a standard, micropillared substrate with nanofilaments (Fig. 2a, right) [6]. The pressure-induced collapse of the standard "micro-Cassie" state now resulted in a Wenzel-like state that has water between the micropillars, and we call it "nano-Cassie" state because there remains a nanoscopic air layer present keeping the wetted solid fraction small. This allowed for a reversible transition back to the original state by a small input of energy in the form of suction. Recently, the Zheng group even reported on fully reversible and spontaneous (= energy input-free) transition on substrates with suitable dual-level roughness (Fig. 2b, right) [7]. The otherwise irreversible wetting transition was shown to be reversible on the dual-level surface.

\section{Fluid dynamics of a bouncing droplet}

The motion and fluid dynamics of droplets on superhydrophobic surfaces can often be both striking and unexpected (see example in Box 1). An exciting area of today's research is in the bouncing of droplets off superhydrophobic surfaces. Typically, the drops spread out nearly axisymmetrically, retract and finally leave the surface (Fig. 3a). In doing so, there is a theoretical lower limit in the required contact time between the drop and the solid. One of the current challenges lies in finding new ways to further reduce the contact time to allow for efficient anti-icing and dropwise condensation applications. By introducing macroscale texture to the substrate, the group of Varanasi managed to break the axisymmetry involved in bouncing (Fig. 3b), resulting in redistribution of liquid mass, and thereby altering the hydrodynamics and reducing contact time by a factor of four compared to what has previously been considered the gold standard [8]. In a clever approach by the Wang group [9], a superhydrophobic surface was made of tapered, nanoflower-coated pillars with characteristic dimensions one order of magnitude larger than the conventional micropillared design. Water drops with high enough inertia were shown to spread out and then leave the surface without retracting (Fig. 3c). This "pancake" bouncing also allowed for a fourfold reduction in the contact time, compared to conventional bouncing. 


\section{Conclusions}

During the last years, great advances have been made in deciphering the physics behind superhydrophobicity. A fundamental understanding of the intricate wetting characteristics is being formed and novel ways to avoid wetting or utilize its characteristics are explored. New exciting research avenues are continuously being created to get one step closer to the superrepellent materials of the future, revealing functions and features that will greatly simplify and improve our everyday lives.

\section{About the authors}

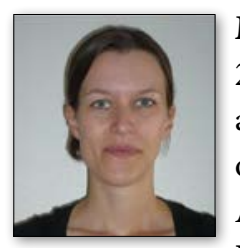

Matilda Backholm received her $\mathrm{PhD}$ in 2015 from McMaster University, Canada, and is currently working as an Academy of Finland postdoctoral researcher at Aalto University.

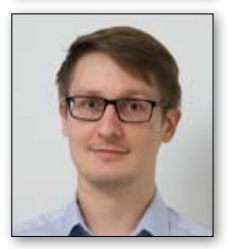

Jaakko V. I. Timonen is professor of the Active Matter group at Aalto University. $\mathrm{He}$ received his $\mathrm{PhD}$ from the same university in 2013 and did postdocs in the Grzybowski and Aizenberg groups at Northwestern University and Harvard, USA.

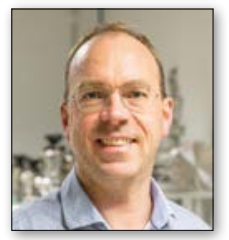

Robin H. A. Ras is professor of the Soft Matter and Wetting group at Aalto University. He received his $\mathrm{PhD}$ in 2003 from the University of Leuven, Belgium, and was awarded an ERC Consolidator grant in 2016.

\section{References}

[1] Purity of the sacred lotus, or escape from contamination in biological surfaces, W. Barthlott and C. Neinhuis, Planta 202, 1 (1997).

[2] Mechanically durable superhydrophobic surfaces, T. Verho, C. Bower, P. Andrew, S. Franssila, O. Ikkala, and R.H.A. Ras, Adv. Mater. 23, 673 (2011).

[3] Moving superhydrophobic surfaces toward real-world applications, X. Tian, T. Verho, and R.H.A. Ras, Science 352, 142 (2016).

[4] How water advances on superhydrophobic surfaces, F. Schellenberger, N. Encinas, D. Vollmer, and H.-J. Butt, Phys. Rev. Lett. 116 , 096101 (2016).

[5] Dry Under Water: Comparative Morphology and Functional Aspects of Air-Retaining Insect Surfaces, A. Balmert, H.F. Bohn, P. Ditsche-Kuru, and W. Barthlott, J. Morphol. 272, 442 (2011).

[6] Reversible switching between superhydrophobic states on a hierarchically structured surface, T. Verho, J.T. Korhonen, L. Sainiemi, V. Jokinen, C. Bower, K. Franze, S. Franssila, P. Andrew, O. Ikkala, and R.H.A. Ras, Proc. Natl. Acad. Sci. USA 26, 10210 (2012).

[7] Monostable superrepellent materials, Y. Li, D. Quéré, C. Lv, and Q. Zheng, Proc. Natl. Acad. Sci. USA 114, 3387 (2017).

[8] Reducing the contact time of a bouncing drop, J.C. Bird, R Dhiman, H.-M. Kwon, and K.K. Varanasi, Nature 503, 385 (2013).

[9] Pancake bouncing on superhydrophobic surfaces, Y. Liu, L. Moevius, X. Xu, T. Qian, J.M. Yeomans, and Z. Wang, Nat. Phys. 10, 515 (2014).

[10] Switchable static and dynamic self-assembly of magnetic droplets on superhydrophobic surfaces, J.V.I. Timonen, M. Latikka, L. Leibler, R.H.A. Ras, and O. Ikkala, Science 341, 253 (2013).

\section{SELF-ASSEMBLING MAGNETIC DROPLETS ON SUPERHYDROPHOBIC SURFACES}

Ferrofluids are colloidal dispersions of magnetic nanoparticles in a carrier liquid. They exhibit a striking coupling between fluidic and magnetic properties. We asked ourselves a question: what kind of new phenomena can be observed if the fascinating features of ferrofluids are combined with the extreme non-wetting of superhydrophobic surfaces?

We tested this experimentally by placing an aqueous ferrofluid droplet on a superhydrophobic surface and subjecting it to a gradually increasing vertical magnetic field [10]. As a result, the shape of the droplet changed from nearly perfectly spherical to elongated along the field direction, until, spontaneously, the droplet split into two smaller droplets (Fig. 4a and Movie youtu.be/ehvFFbFFsjg). This splitting can be understood as the classic Rosensweig instability taking place within the droplet and is driven by the decrease of magnetic energy (at the expense of surface energy). The role of the superhydrophobic surface is to keep the droplets separated, and to allow for low-friction droplet motion to their equilibrium positions. Further increasing the magnetic field can lead to additional splitting events and nearly a hundred droplets can easily be formed. These droplets spontaneously assemble into well-defined clusters due to mutual dipolar repulsion and interaction with the spatially varying global confining magnetic field (Fig. 4b). The magnetic droplets can be used as a model system to study and visualize static and dynamic self-assembly processes.

VFIG.4:Magneticdroplets on superhydrophobic surfaces. (a) Profile of magneticdroplet in a gradually increasing magnetic field (from left to right). (b) Hexagonal cluster of 19 daughter droplets formed by multiple consecutive splitting events from one single mother droplet. The "lattice constant" can be tuned by adjusting the droplet-droplet repulsion and confining external potential. Pictures adapted from [10].

(a)
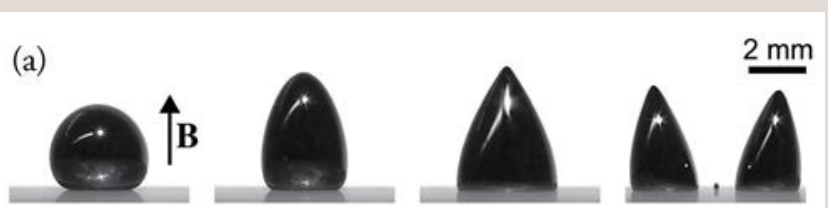

(b)

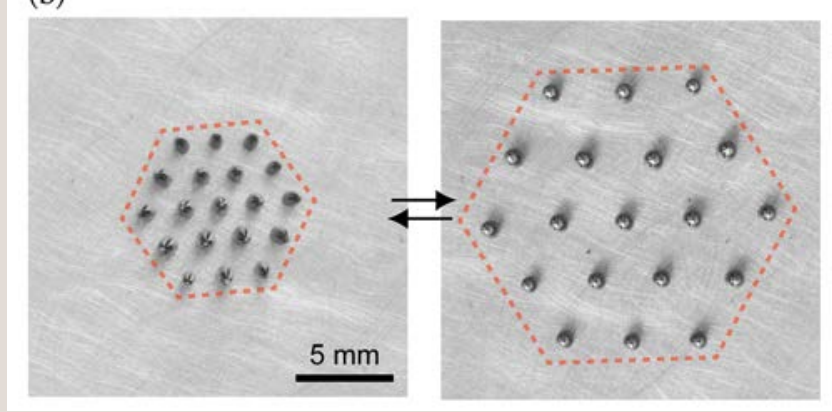

